Apidologie, 1982, 13 (3), 247-263.

\title{
ANALYSE DE L'EQUIPEMENT SENSORIEL ANTENNAIRE DU BOURDON BOMBUS HYPNORUM L.
}

\author{
Caroline FONTA et Claudine MASSON \\ Laboratoire de Neurobiologie Sensorielle de l'Insecte I.N.R.A./C.N.R.S. \\ Station de Recherches sur l'Abeille et les Insectes sociaux \\ 91440 Bures sur Yvette
}

\begin{abstract}
RESUMÉ
Divers auteurs ont mis en évidence le rôle des signaux chimiques dans la communication entre individus dans la colonie de Bourdons. Les sensilla placodea sont des organes sensoriels portés par les antennes et impliqués dans la détection de substances volatiles, en particulier des phéromones. Leur répartition est étudiée ici chez Bombus hypnorum, au moyen de la microscopie électronique à balayage : il n'apparaît aucune différence statistiquement significative entre reine, ouvrière et mâle de cette espèce.

Les autres types de sensilles (olfactives, gustatives, proprioceptrices) sont décrites mais n'ont pas fait l'objet d'une étude quantifiée.
\end{abstract}

\section{INTRODUCTION}

Hyménoptère Apoïdea, de la famille des Apidae, Bombus hypnorum appartient au genre Pyrobombus qui regroupe $1 / 3$ des espèces de Bourdons actuellement connues.

Les bourdons sont classés parmi les insectes sociaux, en effet :

- ils sont organisés en colonies;

- la colonie est composée d'individus apparteriant à plusieurs castes différentes;

- les congénères communiquent entre eux, principalement grâce aux échanges trophallaxiques (trophallaxie indirecte).

Comme pour toute société d'insectes, les odeurs semblent jouer un rôle primordial dans les phénomènes de déterminisme des castes, de régulation sociale, de rencontre des sexes, mais aussi dans de simples processus tels que la localisation du nid et/ou sa défense. 
Si des études éthologiques ont mis en évidence le rôle de signaux chimiques perçus par voie olfactive ou par voie gustative, leur nature, à l'exception des substances de marquage émises par les mâles, est peu (ou pas) connue.

Plusieurs travaux ont effectivement mis en évidence le rôle d'odeurs émises par la reine : phéromone de couvain déclenchant l'incubation chez Bombus vosnesenskii et Bombus edwardsii (HEINRICH, 1974), phéromone royale contribuant à l'accouplement et à la copulation des jeunes reines de Bombus terrestris, Bombus hypnorum, Bombus pratorum (VAN HoNK et col., 1978) mais aussi favorisant un bon développement de la colonie (VAN HoNK et col. 1980). Les travaux de RöSELER (1975, 1977) montrent qu'une production phéromonale régule la fertilité des ouvrières en inactivant leur système endocrine (corpora allata).

Chez certaines espèces (Bombus terrestris), la détermination des castes pourrait être due à l'action d'une phéromone maternelle (RöSELER et RöSELER, 1974; PLOWRIGHT et PENDREL, 1977).

Une autre émission femelle a été mise en évidence, chez les ouvrières de Bombus terrestris (CEDERBERG, 1977) : ces dernières sont capables de déposer et de suivre une odeur de piste.

Le même auteur a assimilé l'acide butyrique, composé majoritaire des glandes mandibulaires de Bombus lapidarius (mâle et femelle), à une substance de défense ( $\mathrm{CE}$ DERBERG, 1977).

Des études comportementales menées chez le mâle, couplées à des analyses chimiques, ont démontré l'existence et la nature d'une phéromone de marquage, produite par la partie céphalique des glandes labiales (KULlENBERG et col., 1970, 1973; BRINGER, 1973; GroTh et col., 1977; ÅGREN et col., 1979). Elle permet au mâle d'établir un territoire et facilite ainsi la rencontre des sexes.

Cette phéromone constitue l'un des facteurs de l'isolement spécifique des bourdons. Elle représente un outil taxonomique important et a été étudiée chez les Bourdons et les Psithyres, leurs commensaux (SvEnsson, 1977, 1980).

Le bilan global des données acquises montre que le domaine à prospecter est vaste, tant dans l'identification des substances émises que dans les mécanismes de reconnaissance de ces odeurs par les insectes. Le travail présenté ici a pour objectif une approche des moyens de détection périphérique antennaire des signaux chimiques chez Bombus hypnorum L. et porte principalement sur les sensilla placodea, sensilles présentes uniquement chez les Insectes Sociaux.

L'équipement sensoriel de l'antenne a été particulièrement étudié chez Apis (DIETZ et Humphreys, 1971; Whitehead et LARSEN, 1976; Standifer et col., 1978...). Les travaux de LACHer et SChNEIDER (1963), KAissling et RENNER (1968), EsSLEN et KAISSLING (1976), confirmant les observations comportementales de von FRISCH (1921) indiquent que les sensilla placodea, présentes en grand nombre sur les 8 segments distaux du funicule de l'Abeille, sont exclusivement dévolues à la fonction olfac- 
tive. De simples études structurales (SLIFER, 1961; SLIFER et SEkhon, 1960, 1961) avaient suggéré que ce type de sensilles était impliqué dans la réception du son (et autres stimuli vibratoires) et la sensibilité aux variations de pression.

Depuis, des études électrophysiologiques chez l'Abeille ont montré que les cellules sensorielles de ces organes récepteurs sont de " type généraliste " (MASson et BROSSUT, 1981) (spectre de réaction large : réponses aux odeurs de fruits, fleurs, acides gras... LACHER, 1964); certaines de ces cellules sont de " type spécialiste " (MASSON et BROSsut, 1981) répondant à la phéromone royale et à l'odeur de la glande de Nasanoff (marquage des sources alimentaires : KAISSLING et RENNER, 1968 : VARESCHI, 1971).

On a également remarqué que les s. placodea pouvaient présenter des aspects différents (Dietz et Humphreys, 1971). Chez Apis, ArNold et MAsson (1981) ont montré l'existence de 2 types discernables, apparaissant de façon différentielle du cours du développement, et qui pourraient être en relation avec la modification de l'environnement odorant de l'insecte. Cet aspect "âge " n'a pas été considéré ici; seules les antennes des individus adultes ont été analysées.

\section{MATÉRIEL ET METHODES}

\section{Matériel biologique}

Les Bombus hypnorum utilisés proviennent d'un élevage maintenu à la Station de Recherches sur l'Abeille et les Insectes Sociaux de Bures-sur-Yvette.

L'étude a été faite sur des insectes provenant principalement d'une même colonie, mais la fourniture lente et aléatoire en matériel biologique de celle-ci (mauvaises conditions climatiques en 1981, attaques d'Aphomia sociella, Lépidoptère Pyralidae) a dû être compensée par l'utilisation d'une autre colonie.

\section{Techniques: préparation du matériel pour l'étude en microscopie électronique à balayage}

Les antennes sont prélevées sur les insectes vivants décapités, nettoyées avec un pinceau fin puis séchées à l'air (pour les antennes d'ouvrières et de mâles). Les fondatrices, prélevées alors qu'elles étaient encore en torpeur, avaient les antennes recouvertes de matériau d'hibernation et donc étaient impropres à une observation directe. Dans ce cas, les antennes ont été trempées quelques secondes dans l'alcool éthylique à $70^{\circ}$, afin de parfaire le nettoyage, et séchées à l'air.

La base des antennes est fixée sur le porte-objet au moyen d'une goutte de laque d'argent (afin d'assurer un bon contact électrique avec le support). La préparation est métallisée à l'or-palladium (évaporation sous vide).

Les observations ont été effectuées sur microscope électronique à balayage CAMECA 07, sous une tension de $20 \mathrm{kV}$.

Le recensement des sensilles est effectué à partir des clichés photographiques (grossissement 500) qui ont servi à reconstituer l'organisation spatiale de l'ensemble des antennes droite et gauche de chaque caste et sexe.

Compte tenu de la relative fixité de chaque montage d'antenne, l'analyse des diverses faces d'une même antenne a nécessité l'examen d'antennes appartenant à des individus différents.

Les résultats présentés ci-dessous ne sont pas exhaustifs. L'étude a été volontairement limitée à la face supérieure de l'antenne, plus riche en équipement sensoriel. 
Pour l'analyse statistique, une " aire de comptage " a été délimitée et choisie dans la portion d'antenne apparaissant le plus nettement à l'observation, et qui correspond à $1 / 6$ de la surface totale de l'antenne, celle-ci étant assimilée à un cylindre (cf. Annexe).

Afin de pouvoir effectuer des comparaisons entre les antennes, nous avons éliminé le facteur taille de l'antenne, en raisonnant sur la densité des sensilles.

La méthode statistique utilisée est le " test t par paires " qui permet une comparaison entre segments homologues de deux antennes.

\section{RÉSULTATS}

L'antenne de Bombus hypnorum présente une forte densité de sensilles, plus diversifiées, quant à leur morphologie externe, sur la face supérieure (ou dorsale) que sur la face inférieure (ou ventrale) de l'antenne, aussi bien chez la reine, l'ouvrière que le mâle.

\section{Les articulations (cf. Fig. 1)}

Au niveau de l'articulation basale de l'antenne sur la capsule céphalique, on trouve sur la rotule, à la base du scape, des propriocepteurs (" hair-plates "). Ce même type de sensilles est présent, au niveau des faces externe et interne du scape, à l'articulation du scape et du pédicelle. Cet équipement est comparable à celui décrit par Masson (1972) chez la fourmi Camponotus et chez laquelle il a été démontré (MASson, 1973) que ces structures proprioceptrices assuraient une autorégulation de la posture antennaire (cf. Fig. $1:$ A, B, C).

Au niveau de la bordure distale du pédicelle, une couronne de sensilles campaniformes pourrait être adaptée à la réception de stimulations multidirectionnelles, en association avec les terminaisons des structures chordotonales de l'organe de Johnston, situées dans la membrane entre le pédicelle et le premier article du funicule (MAsson, 1973; Masson et Gabouriaut, 1973). Une étude de ce type, purement morphologique, ne permet pas de conclure sur la fonction éventuelle de ces structures; une analyse en microscopie électronique par transmission serait au moins nécessaire (cf. Fig. 1: D).

\section{Le flagelle (cf. Fig. 2)}

\subsection{Sensilles olfactives}

Les articles du flagelle sont richement équipés en sensilles olfactives : sensilla placodea, sensilla trichodea, sensilla basiconica, sensilla coeloconica, sensilla ampullacea (SCHNEIDER et STEINBRECHT, 1968). Une estimation de la répartition de ces deux derniers types de sensilles semble montrer l'existence d'un gradient croissant vers l'extrémité du flagelle chez la fourmi Camponotus vagus (Masson, 1973); le même type d'observation a été fait pour des sensilla basiconica chez la fourmi Acromyrmex octospinosus (Delabie et Masson. 1982). 

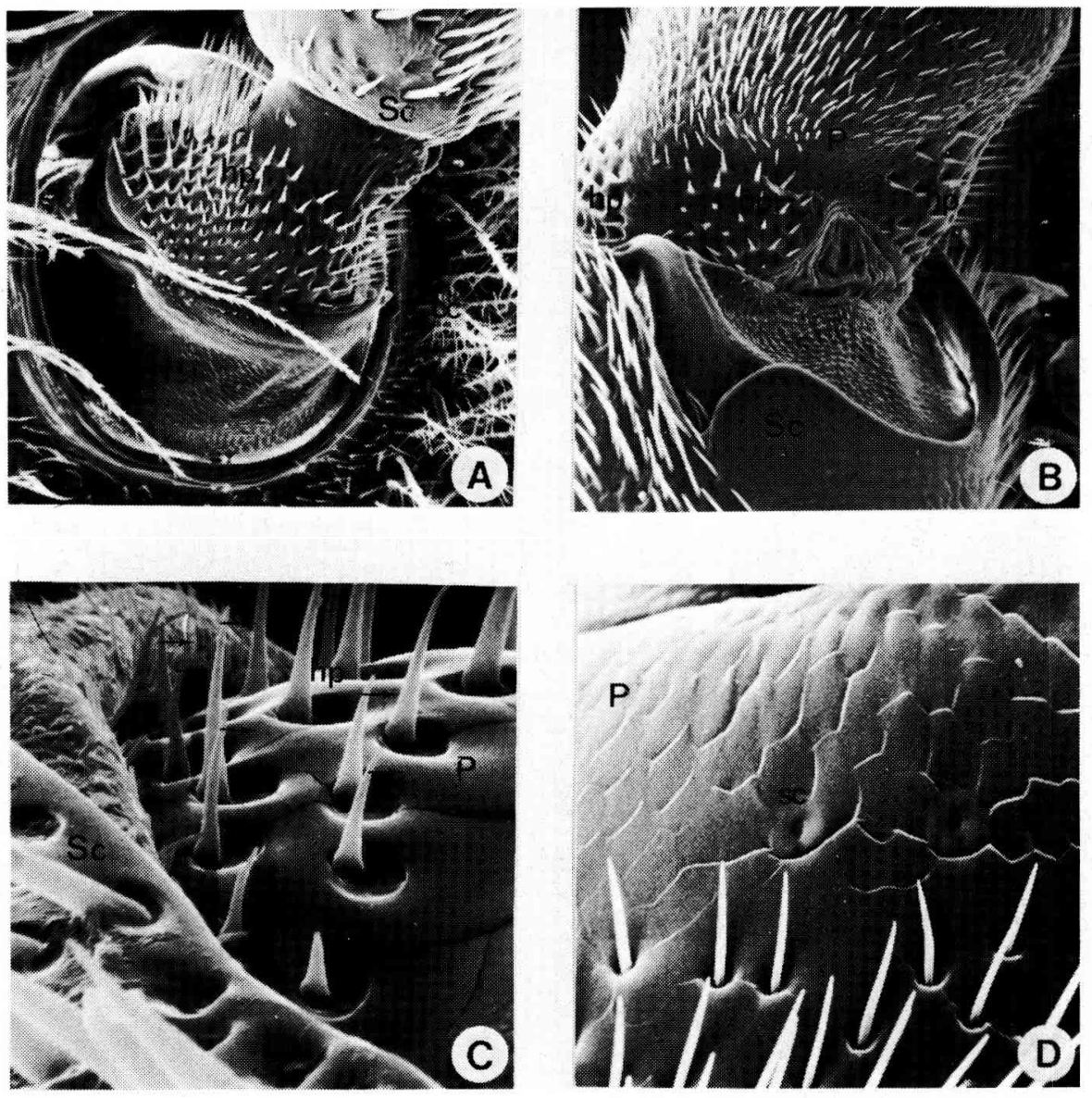

FIG. 1. - Équipement sensoriel de l'antenne au niveau des articulations.

(Clichés obtenus au microscope électronique à balayage.)

A. Articulation basale de l'antenne sur la capsule céphalique (c.c.) (fondatrice). On peut observer l'équipement en structures de type " hair-plates" (h.p.) de la rotule d'articulation (r) du scape (Sc.), véritables récepteurs de position $(\times 210)$.

B. Articulation entre le scape (Sc.) et le pédicelle $(P)$ de l'antenne (fondatrice). Les groupes de "hairplates " (h.p.) situés en position externe en contrôlent l'ouverture et ceux situés en position interne en contrôlent la fermeture $(\times 320)$.

C. "Hair-plates " (h.p.) de l'articulation scape-pédicelle (Sc.-P) face externe (ouvrière). Le déplacement de la soie $(\rightarrow)$ entraîne une déformation de la terminaison nerveuse sous-jacente et la naissance d'un influx nerveux qui sera transmis au cerveau (deutocérébron) ( $\times 2125$ ).

D. Sensilles campaniformes (s.c.) situées à la partie distale du pédicelle $(P)$ (fondatrice) $(\times 1530)$.

FIG. 1. - Sensory equipment at the antennal articulations.

(Scanning Electron Microscopy, S.E.M.)

A. Antennal basal articulation on the cephalic capsule (c.c.) (queen). "Hair-plates" type structures (h.p.) can be seen on the scape (Sc.) articulation rotula (r); they are real position receptors $(\times 210)$.

B. Articulation between the scape ( $\mathrm{Sc}$.) and the pedicel $(\mathrm{P})$ of the antenna (queen). Opening is controlled by outside " hair-plates " (h.p.) groups, closing by inside " hair-plates " groups (x 320 ).

C. Outer scape-pedicel (Sc.-P) articulation " hair-plates " (h.p.) (worker). Seta moving $(\rightarrow$ ) involves an underlying nerve ending distorsion and gives rise to the nerve impulse which will be conveyed to the brain (deutocerebrum) $(\times 2,125)$.

D. Sensilla campaniformia of the pedicel (P) distal part (queen) $(\times 1,530)$. 

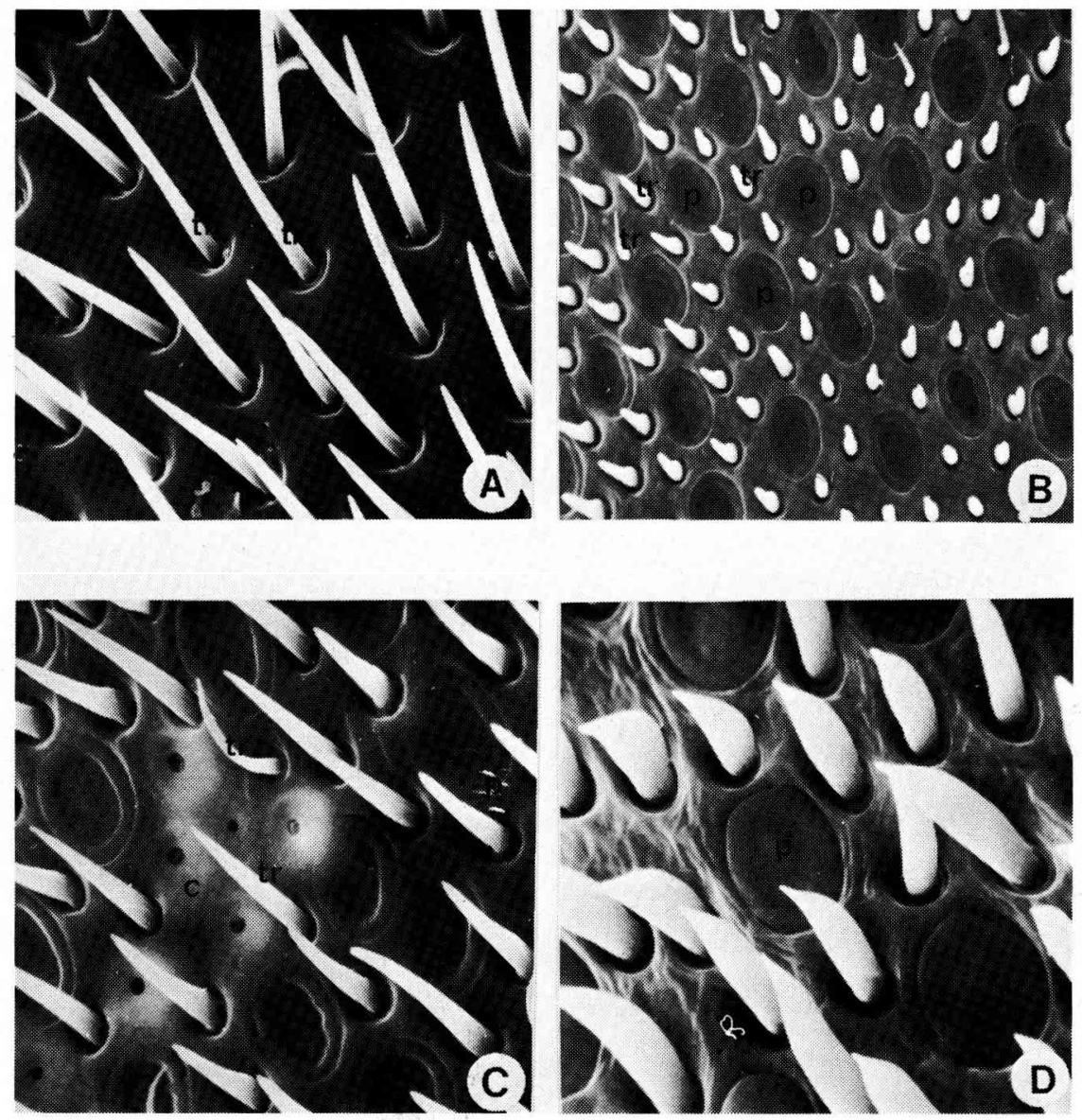

FIG. 2. - Équipement du flagelle de l'antenne en sensilles olfactives.

(Clichés obtenus au microscope à balayage.)

A. Face ventrale (ou inférieure) de l'antenne : on n'observe que des sensilla trichodea (tr.) (ouvrière) $(\times 2125)$

B. Équipement sensoriel de la face dorsale (ou supérieure) de l'antenne chez le mâle (segment III) : les sensilla placodea $(\mathrm{p})$ et les sensilla trichodea $(\mathrm{tr})$ constituent un réseau régulier $(\times 1020)$.

C. Sensilles olfactives : s. placodea (p), s. coeloconica (c) (ou s. ampullacea), s. trichodea (tr), telles qu'on les observe sur la face dorsale de l'antenne du mâle (XIe article) $(\times 2125)$.

D. Sensilla placodea (face dorsale de l'antenne, $\mathrm{II}^{\mathrm{e}}$ article, ouvrière) $(\times 2125)$.

FIG. 2. - Olfactory antennal sensory equipment (S.E.M.).

A. Antennal ventral (inferior) side : only sensilla trichodea $(\mathrm{tr})$ can be seen (worker) $(\times 2,125)$.

B. Antennal dorsal (superior) side of a male sensory equipment (segment III) : sensilla placodea $(\mathrm{p})$ and sensilla trichodea $(\mathrm{tr})$ build up a regular network $(\times 1,020)$.

C. Olfactory sensilla : s. placodea (p), s. coeloconica (c) (or s. ampullacea) s. trichodea (tr), as they can be seen on the dorsal side of the male antenna (article XI) $(\times 2,125)$.

D. Sensilla placodea (antennal dorsal side, article II, worker) $(\times 2,125)$. 
L'étude des sensilles olfactives n'a été quantifiée que pour les sensilla placodea, sensilles dont nous avons signalé l'importance dans la perception des signaux olfactifs et principalement phéromonaux, dans la société d'Apis mellifica.

Le recensement de ce type de sensilles chez la fondatrice, l'ouvrière et le mâle est reporté respectivement dans les tableaux $1,2,3$.

TABL1. - Répartition des sensilia placodea chez la fondatrice.

(Face supérieure de l'antenne) : résultats bruts.

$\mathrm{N}$ : nombre de sensilles sur la surface antennaire étudiée.

$\mathrm{d}$ : densité (nombre de s. placodea par millimètre carré sur la surface étudiée).

Se : surface antennaire exploitée $10^{-6} \mathrm{~mm}^{2}$.

ST : surface antennaire totale $10^{-6} \mathrm{~mm}^{2}$.

TABL. 1. - Spatial distribution of the sensilla placodea for the queen.

(Antennal superior side) : raw data.

$\mathrm{N}$ : sensilla number on the studied antennal area.

$\mathrm{d}$ : density (s. placodea number $/ \mathrm{mm}^{2}$ studied area).

Se : exploited antennal area $10^{-6} \mathrm{~mm}^{2}$.

ST : total antennal area $10^{-6} \mathrm{~mm}^{2}$

\begin{tabular}{|c|c|c|c|c|c|c|c|c|}
\hline \multirow{3}{*}{$\begin{array}{c}\text { Article } \\
\text { antenaire } \\
\text { Antennal } \\
\text { article }\end{array}$} & \multicolumn{8}{|c|}{$\begin{array}{l}\text { Fondatrice } \\
\text { Foundress }\end{array}$} \\
\hline & \multicolumn{4}{|c|}{$\begin{array}{l}\text { Antenne droite } \\
\text { Right antenna }\end{array}$} & \multicolumn{4}{|c|}{$\begin{array}{l}\text { Antenne gauche } \\
\text { Left antenna }\end{array}$} \\
\hline & $\mathrm{N}$ & d & $\mathrm{Se}$ & ST & $\mathbf{N}$ & d & $\mathrm{Se}$ & ST \\
\hline $\begin{array}{c}\text { I } \\
\text { II } \\
\text { III } \\
\text { IV } \\
\text { V } \\
\text { VI } \\
\text { VII } \\
\text { VIII } \\
\text { IX } \\
\text { X } \\
\text { XI }\end{array}$ & $\begin{array}{r}0 \\
48 \\
153 \\
160 \\
171 \\
163 \\
182 \\
157 \\
174 \\
208\end{array}$ & $\begin{array}{l}1411 \\
4260 \\
4420 \\
4358 \\
4370 \\
4935 \\
3911 \\
4045 \\
3530\end{array}$ & $\begin{array}{l}34016 \\
35916 \\
36192 \\
39236 \\
37304 \\
36876 \\
40140 \\
43020 \\
58928^{a}\end{array}$ & 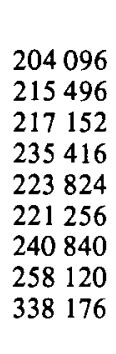 & $\begin{array}{r}0 \\
42 \\
140 \\
151 \\
153 \\
160 \\
166 \\
175 \\
174 \\
169\end{array}$ & $\begin{array}{lll}1 & 141 \\
3 & 320 \\
3 & 574 \\
3 & 807 \\
3 & 893 \\
4 & 253 \\
4 & 354 \\
4 & 469 \\
3 & 739\end{array}$ & $\begin{array}{ll}36 & 836 \\
42 & 172 \\
42 & 252 \\
40 & 188 \\
41 & 104 \\
39 & 028 \\
40 & 192 \\
38 & 936 \\
45 & 200^{b}\end{array}$ & $\begin{array}{ll}221 & 016 \\
253 & 032 \\
253 & 512 \\
241 & 128 \\
246 & 624 \\
234 & 168 \\
241 & 152 \\
233 & 616 \\
258 & 744\end{array}$ \\
\hline & \multicolumn{4}{|c|}{$\bar{x}_{d}=3916$} & \multicolumn{4}{|c|}{$\bar{x}_{d}=3617$} \\
\hline
\end{tabular}

$a=16,67 \%$ ST

$\mathrm{b}=17,47 \% \mathrm{ST}$

A partir des données obtenues, nous avons, dans une première étape, comparé l'antenne droite et l'antenne gauche chez la reine, l'ouvrière et le mâle, au moyen d'un test $t$ par paires (cf. Tabl. $4 \mathrm{~A}$ ).

Les deux antennes d'un même "individu " ne sont pas significativement différentes pour un risque $\alpha$ de $5 \%$ et même de $1 \%$. Ces résultats suggèrent qu'il ne doit pas exister de latéralisation dans la perception des odeurs par les sensilla placodea. 


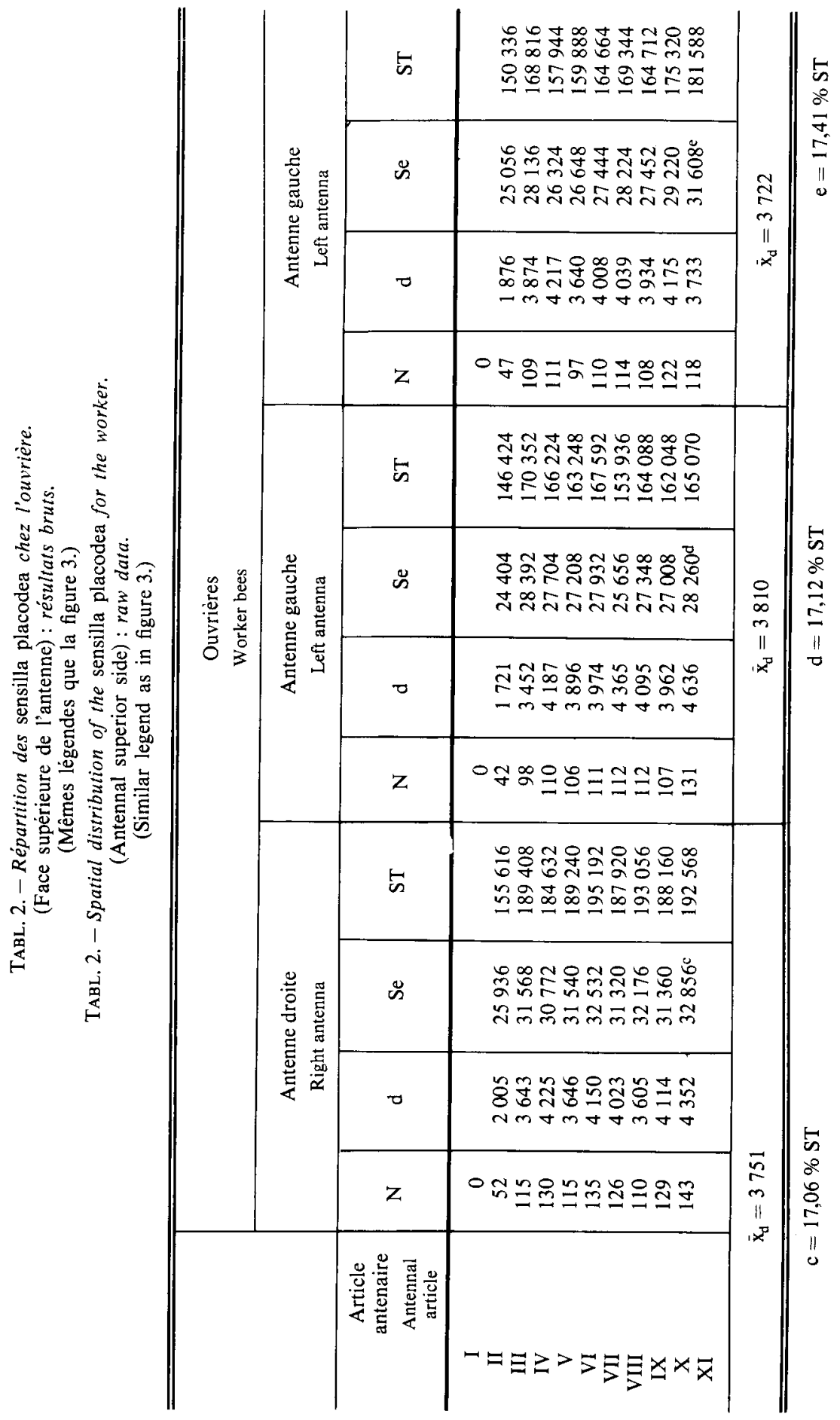


TABL. 3. - Répartition des sensilla placodea chez le mâle.

(Face supérieure de l'antenne) : résultats bruts.

(Mêmes légendes que la figure 3.)

TABL. 3. - Spatial distribution of the sensilla placodea for the male.

(Antennal superior side) : raw data.

(Similar legend as in figure 3.)

\begin{tabular}{|c|c|c|c|c|c|c|c|c|}
\hline \multirow[b]{3}{*}{$\begin{array}{c}\text { Article } \\
\text { antenaire } \\
\text { Antennal } \\
\text { article }\end{array}$} & \multicolumn{8}{|c|}{$\begin{array}{l}\text { Mâle } \\
\text { Male }\end{array}$} \\
\hline & \multicolumn{4}{|c|}{$\begin{array}{l}\text { Antenne droite } \\
\text { Right antenna }\end{array}$} & \multicolumn{4}{|c|}{$\begin{array}{c}\text { Antenne gauche } \\
\text { Left antenna }\end{array}$} \\
\hline & $\mathbf{N}$ & $\mathrm{d}$ & $\mathrm{Se}$ & ST & $\mathbf{N}$ & $\mathrm{d}$ & $\mathrm{Se}$ & ST \\
\hline $\begin{array}{r}\text { I } \\
\text { II } \\
\text { III } \\
\text { IV } \\
\text { V } \\
\text { VI } \\
\text { VII } \\
\text { VIII } \\
\text { IX } \\
\text { X } \\
\text { XI }\end{array}$ & $\begin{array}{r}0 \\
40 \\
115 \\
135 \\
137 \\
141 \\
150 \\
126 \\
127 \\
123 \\
164\end{array}$ & $\begin{array}{lll}1 & 325 \\
2 & 927 \\
3 & 295 \\
3 & 358 \\
3 & 583 \\
3 & 754 \\
3 & 422 \\
3 & 608 \\
3 & 572 \\
3 & 931\end{array}$ & $\begin{array}{l}30184 \\
39288 \\
40968 \\
40800 \\
39356 \\
39960 \\
36824 \\
35200 \\
34436 \\
41716^{f}\end{array}$ & $\begin{array}{l}181104 \\
235728 \\
245804 \\
244800 \\
236136 \\
239760 \\
220944 \\
211200 \\
206616 \\
229540\end{array}$ & $\begin{array}{r}0 \\
37 \\
132 \\
143 \\
142 \\
145 \\
154 \\
135 \\
147 \\
124 \\
163\end{array}$ & $\begin{array}{ll}1 & 247 \\
3 & 272 \\
3 & 399 \\
3 & 392 \\
3 & 499 \\
4 & 044 \\
3 & 722 \\
4 & 006 \\
3 & 671 \\
4 & 338\end{array}$ & $\begin{array}{lll}29 & 680 \\
40 & 340 \\
42 & 068 \\
41 & 868 \\
41 & 444 \\
38 & 080 \\
36 & 272 \\
36 & 692 \\
33 & 776 \\
37 & 572 \\
\end{array}$ & 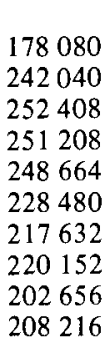 \\
\hline & \multicolumn{4}{|c|}{$\bar{x}_{d}=3278$} & \multicolumn{4}{|c|}{$\bar{x}_{d}=3459$} \\
\hline
\end{tabular}

TABL. 4. - Répartition des sensilla placodea : données statistiques.

TABL. 4 A. - Résultats statistiques de la comparaison entre les antennes droite et gauche chez la fondatrice, l'ouvrière et le mâle (effectuée sur les données brutes).

TABL. 4. - Spatial distribution of the sensilla placodea : statistical results.

TABL. 4 A. - Statistical results for the comparison between right and left antennae for the queen,

the worker and the male (calculated with the raw data).

\begin{tabular}{l|c|c|c|c|c}
\hline \hline & t obs. & nbre ddl & $\mathrm{t}$ lu $\alpha=5 \%$ & $\mathrm{t} l \mathrm{u} \alpha=1 \%$ & $\begin{array}{c}\text { Signification } \\
\text { Significance }\end{array}$ \\
\hline $\begin{array}{l}\text { Fondatrice } \\
\text { Foundress } \\
\begin{array}{l}\text { Ouvrière } \\
\text { Worker bee }\end{array}\end{array}$ & 1,07 & 8 & 2,306 & 3,355 & N.S. \\
$\begin{array}{l}\text { Mâle } \\
\text { Male }\end{array}$ & 3,13 & 9 & 2,306 & 3,355 & N.S. \\
\hline \hline
\end{tabular}


TABL. 4 B. - Résultats statistiques de la comparaison de l'équipement en sensilla placodea, entre la fondatrice et l'ouvrière (calculs effectués sur les moyennes calculées).

TABL. 4 B. - Statistical results for the comparison between queen's and worker's sensilla placodea equipment

(calculated with the calculated means).

\begin{tabular}{c|c|c|c|c}
\hline \hline t obs & nbre ddl & $\mathrm{t}$ lu $\alpha=5 \%$ & $\mathrm{t}$ lu $\alpha=1 \%$ & $\begin{array}{c}\text { Signification } \\
\text { Significance }\end{array}$ \\
\hline 0,039 & 8 & 2,306 & 3,355 & N.S. \\
\hline
\end{tabular}

Cette vérification étant faite, il est alors possible de calculer une densité moyenne de sensilla placodea par segment et de tracer l'histogramme correspondant à la densité moyenne de sensilles pour chaque article antennaire chez la reine, l'ouvrière et le mâle (cf. Fig. 3).

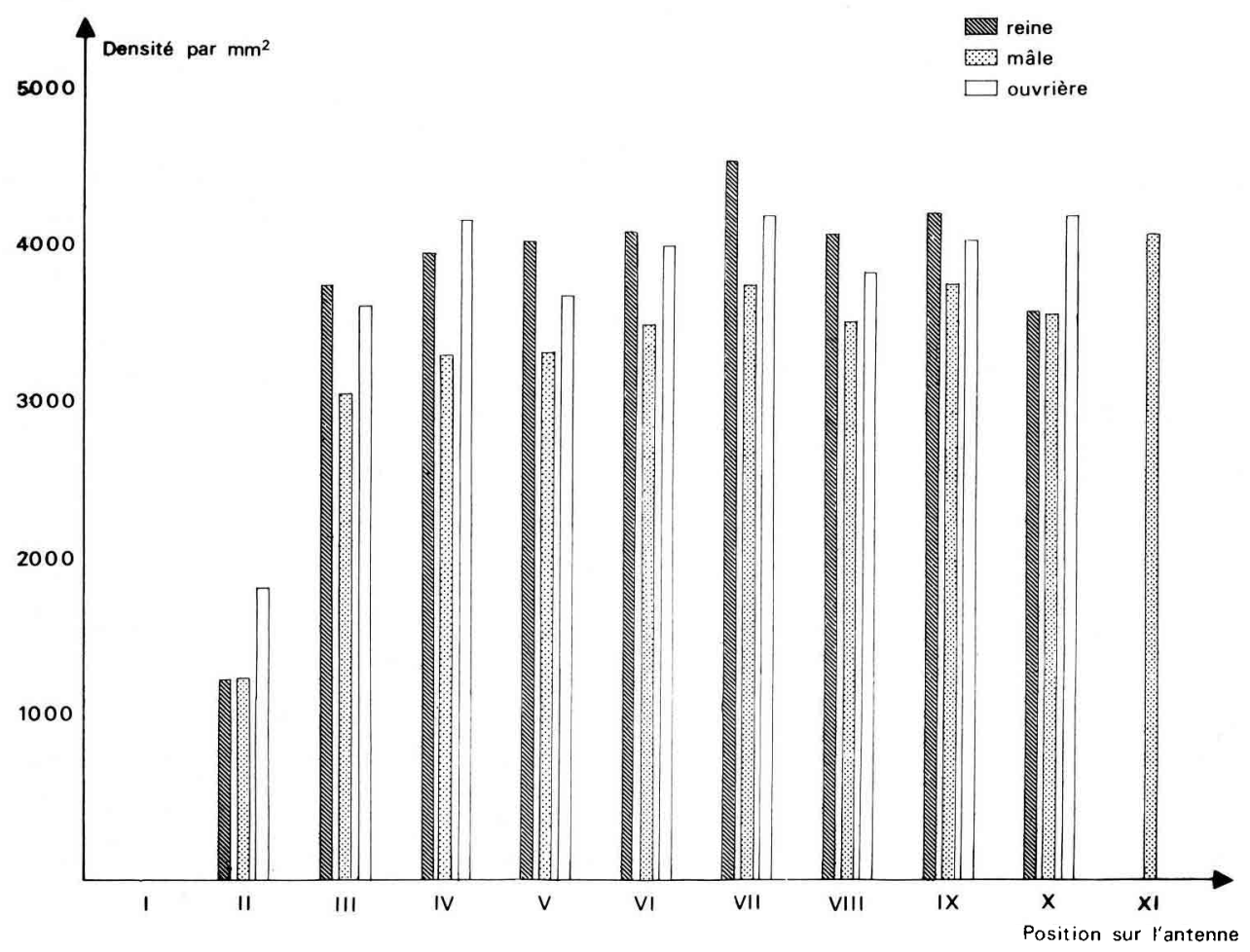

Fig. 3. - Densité moyenne de sensilla placodea par article du flagelle de l'antenne. en abscisse : article du flagelle;

en ordonnée : nombre de sensilla placodea par millimètre carré de surface antennaire exploitée.

FIG. 3. - Average density of sensilla placodea/antennal flagellum article.

$\mathbf{x}$ - axis : flagellum article;

$\mathrm{y}$ - axis : sensilla placodea number $/ \mathrm{mm}^{2}$ exploited antennal area. 

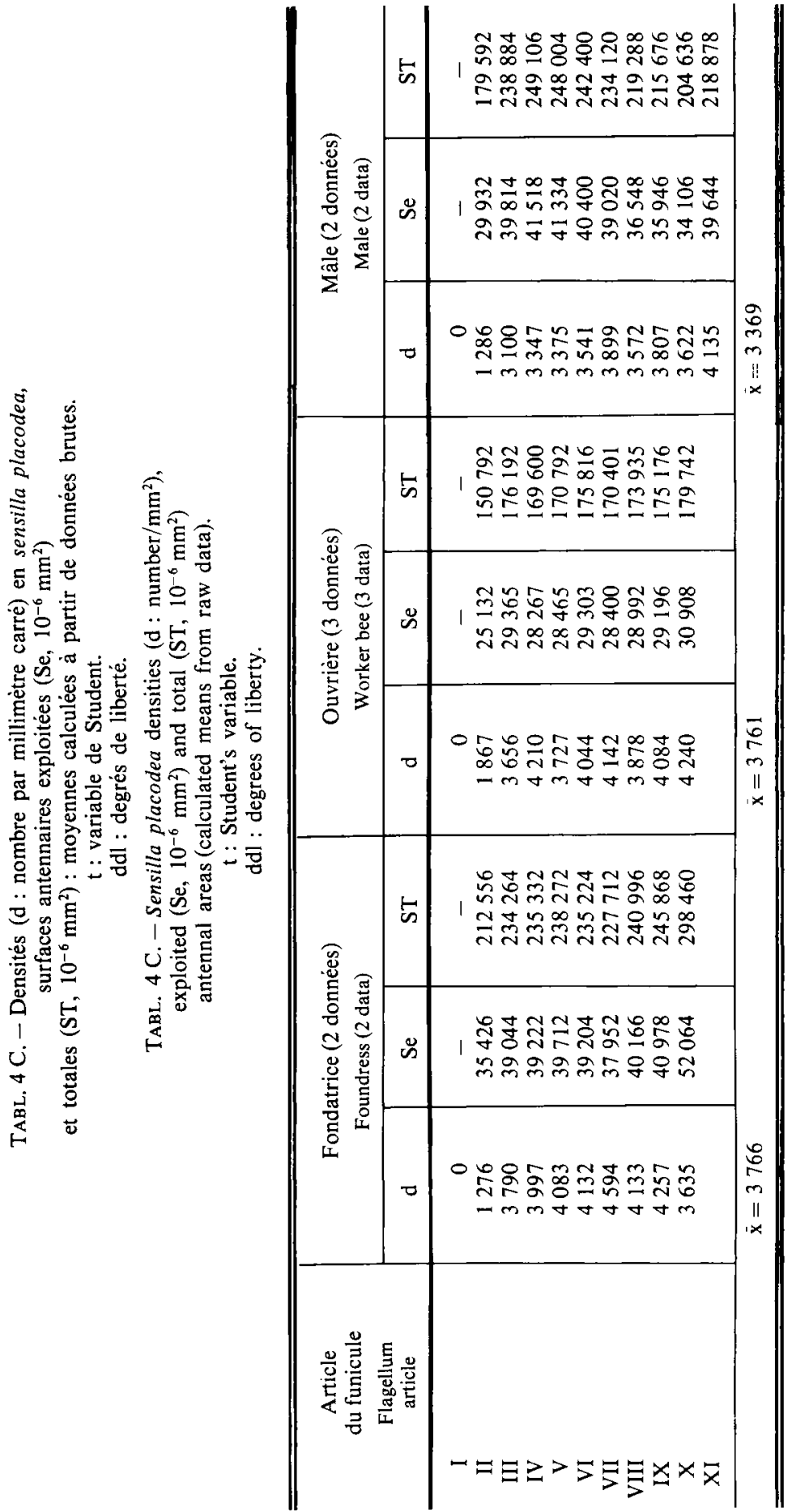
Dans l'état actuel des données recueillies, étant donné la taille de l'échantillon, l'angle de prise de vue des photographies variable (ce qui se répercute directement sur l'estimation de la surface antennaire), le grossissement trop faible pour la distinction nette de certains détails, il est délicat d'effectuer une analyse statistique. De plus, le mâle possédant un article antennaire supplémentaire, le test $t$ ne permet donc ici que de comparer des individus de même sexe.

Aucune différence significative n'existe entre la reine et l'ouvrière pour un risque $\alpha$ de $1 \%$ (cf. Tabl. 4 B).

Pour les trois types d'individus étudiés, le $1^{\text {er }}$ segment du flagelle est dépourvu de sensilla placodea, le $2^{\mathbf{e}}$ segment est nettement moins équipé que les suivants en ce type de sensilles (cf. Tabl. $4 \mathrm{C}$ ).

Si entre le $3^{e}$ et le $10^{e}$ segment la distribution des sensilla placodea chez l'ouvrière fluctue entre 3656 et 4240 sensilla placodea par millimètre carré, on peut remarquer chez les individus sexués une évolution parallèle : il existe un double mode de distribution des sensilles entre les $3^{e}$ et $10^{e}$ segments au niveau du segement VII (4 594 et 3899 placodea par millimètre carré respectivement chez reine et mâle) et du segment IX (4 257 et 3807 placodea par millimètre carré respectivement chez reine et mâle). Toutefois, chez le mâle, c'est le dernier article antennaire qui est le plus richement pourvu (article XI) : 4135 placodea par millimètre carré.

\subsection{Sensilles gustatives}

Principalement sur les articles distaux du flagelle (mais plus régulièrement réparties chez le mâle), et en petit nombre, sont distribuées des soies dont les caractéristiques morphologiques, comparées aux données de la littérature, suggèrent qu'elles appartiennent à la classe des sensilles gustatives. Chez d'autres Hyménoptères sociaux, notamment chez les fourmis, Masson $(1973,1974)$ a décrit des structures semblables en microscopie électronique à balayage et par transmission, et en a démontré la fonction gustative par enregistrements électrophysiologiques (MASSON, 1973).

\section{DISCUSSION - CONCLUSION}

Compte tenu de la dimension de l'échantillon associée à la difficulté des observations, il parait délicat de vouloir effectuer une comparaison entre les deux sexes dans l'équipement sensoriel antennaire des Bourdons.

Chez Apis, il existe un dimorphisme notable entre l'ouvrière et mâle dans le nombre relatif de sensilles de chaque type et le nombre total de cellules sensorielles; pour une surface antennaire 2 fois plus grande, le mâle possède 5 fois plus de cellules sensorielles que l'ouvrière (EsSLEN et KaISSLING, 1976).

On sait que, chez l'abeille, l'accouplement est contrôlé par la phéromone royale perçue par les mâles. Chez le Bourdon, le comportement, lors de la rencontre des sexes 
est plus complexe. Le mâle émet une substance phéromonale de marquage de territoire qui a un rôle dans l'attraction de la femelle, mais cette dernière produit elle aussi une phéromone permettant un comportement d'accouplement et de copulation (VAN HONK et col., 1978).

La similarité de l'équipement en sensilla placodea entre reine et ouvrière ne paraît pas à première vue étonnante, les 2 castes accomplissant certaines tâches identiques (butinage, soins au couvain...). Mais cette étude n'indique pas le type de réponses des cellules sensorielles aux arômes floraux, aux odeurs phéromonales.

Il peut être dangereux de vouloir établir une relation entre la structure externe et la fonction de l'équipement sensoriel. Une véritable discrimination entre les castes ne peut être révélée que par l'étude électrophysiologique unitaire des cellules sensorielles. Le travail exposé ci-dessus est destiné à préparer dans ce sens le développement de cette recherche. Chez le Bourdon, cette approche ne présentant pas à notre connaissance d'antécédents, a été menée en comparaison à d'autres Hyménoptères, en particulier à l'Abeille. Les Hyménoptères sociaux ont un spectre d'interactions comportementales qui, dans leur totalité, dépassent celles rencontrées dans les espèces présociales. Un haut degré d'organisation est maintenu pour intégrer de façon optimale les activités des membres de la colonie.

Les signaux chimiques sont les principaux vecteurs gouvernant les interactions comportementales spécifiques entre les individus. Le danger présent pourrait être une assimilation du cas Bourdon au type Abeille. Sans vouloir établir de lien phylogénétique, il faut cependant noter entre les deux types de sociétés des différences attribuables peut-être à la différence de potentialité de l'insecte dans la production et dans la perception du signal chimique, à l'importance relative du rôle de la communication chimique par rapport aux autres moyens tels que les sens tactiles, visuels...

Les différences comportementales observées peuvent être utilisées, en particulier dans les services de pollinisation.

\section{REMERCIEMENTS}

Les auteurs tiennent à remercier Mme Gulllaumin, ingénieur au Service de Microscopie Électronique du Laboratoire d'Évolution des Êtres Organisés (Université de Paris VI) pour son aide efficace aussi bien pour les observations que pour les clichés photographiques. Iis remercient également le $\mathrm{Dr} \mathrm{A}$. PouVREAU pour son aide dans la fourniture du matériel biologique. 


\section{ZUSAMMENFASSUNG

\author{
ANALYSE DER AUSSTATTUNG DER ANTENNEN \\ DER HUMMEL BOMBUS HYPNORUM L. MIT SINNESORGANEN
}

Die Hummel, ein soziales Insekt, steht mit seinen Artgenossen mittels chemischer Signale in Verbindung. Verschiedene flüchtige Sekrete scheinen für die Entwicklung der Kolonie bestimmend zu sein (Determinierung der Kasten, soziale Regulation, Anlockung der Geschlechtstiere).

Es ist wichtig, den Mechanismus der Erkennung dieser Substanzen durch das Insekt und die erste Etappe des Wahrnehmungsvorganges kennen zu lernen. Die Sensilla placodea sind Geruchsrezeptoren, deren Nervenzellen in besonderem Masse auf Pheromon-Gerüche ansprechen.

Eine Studie von REM-Aufnahmen ergab eine quantitative Aufstellung der Ausstattung der Antennen von Bombus hypnorum mit Sensilla placodea (Tab. 1, 2, und 3 im Anhang). Das Histogramm in Abb. 3 stellt die mittlere Dichte der Sensilla placodea für jedes Antennenglied von Tieren der drei Kasten des Volkes dar. Es lässt sich kein statistisch signifikanter Unterschied zwischen Königin, Arbeiterin und Drohn nachweisen (Tab. 4). Diese Ergebnisse werden mit denen einer analogen Untersuchung an Apis verglichen.

Ausserdem wird eine Schätzung der Verteilung anderer Typen von Geruchssensillen (trichodea, basiconica, coeloconica, ampullacea) sowie von Geschmacks- und Propriorezeptoren (Haarfeldern) gegeben (Abb. 1, 2).

\section{SUMMARY}

ANALYSIS OF THE ANTENNAL SENSILLA EQUIPMENT OF THE BUMBLEBEE

Bumblebees, social insects, communicate with their congeners by means of chemical cues. Various volatile compounds seem determinative for the development of the colony (castes determination, social regulation, mating...).

It is important to know the identification mechanisms of the substances by the insect and the first step of this study is their perception. Sensilla placodea are olfactory receptors, nervous cells of which are specially adapted to detect the odorant pheromones.

A study of scanning electron microscopy (S.E.M.) slides mountings allowed us to quantify the antennal sensilla placodea equipment of Bombus hypnorum (Tabl. 1, 2, 3, Annexe). The histogramm on the figure 3 shows the sensilla placodea average density on each antennal article for the three kinds of the colony members. No statistically significant difference seems to exist between queen, worker and male (Tabl. 4). These results are compared to those got by a similar work in Apis.

A spatial distribution assessment for the other types of olfactory sensilla (trichodea, basiconica, coeloconica, ampullacea), gustatory sensilla and proprioceptors is also presented (Fig. 1, 2).

\section{BIBLIOGRAPHIE}

Ågren L., Cederberg B., Svensson Bo. G., 1979. - Changes with age in ultra-structure and Pheromone content of male labial glands in some Bumble bee species (Hymenoptera, Apidae), Zoon, $7: 1$-14.

ARNold G., Masson C., 1981. - Evolution, en fonction de l'âge, de la structure externe des sensilles olfactives de l'antenne de l'ouvrière d'abeille Apis mellifica L. C.R. A cad. Sc. Paris, Série III, t. 292 : 681-686

BrINGER B., 1973. - Territorial flight of Bumble bee males in coniferous forest in the northernmost part of the island of Öland. Zoon suppl., $1: 15-22$. 
Cederberg B., 1977. - Chemical basis for defense in Bumble bees. Proc. 8th. Int. Congr. I.U.S.S.I. $1977: 77$.

CEDerberg B., 1977. - Evidence for trail marking in Bombus terrestris workers (Hymenoptera, Apidae). Zoon, 5 : 143-146.

Delabie J., Masson C., 1982. - Etude préliminaire de l'équipement sensoriel antennaire d'Acromyrmex octospinosus in Attini $\mathrm{n}^{\circ} 12$.

DiETz A., Humphreys W. J., 1971. - Scanning electron microscopic studies of antennal receptors of the worker Honey Bee, including sensilla campaniformia. Ann. Entomol. Soc. Am. 64 (4) : 919-925.

Esslen J., Kaissling K. E., 1976. - Zahl und Verteilung antennaler Sensillen bei der Honigbiene ( $A$ pis mellifera L.). Zoomorphologie, $83: 227-251$.

FrISCH K. von (1921) cité in FrISCH K. von, 1967. - The dance language and orientation of Bees. Cambridge (Mass.) Harvard University Press. XIV + 566 p., Fig.

Groth I., Appelgren M., Bergstrom G., 1977. - Techniques of analysis and results from work with volatile messengers in plants and insects. Proc. 8th Int. Congr. I.U.S.S.I. 1977 : 302.

HeINRICH B., 1974. - Pheromone induced brooding behavior in Bombus vosnesenskii and B. edwardsii (Hymenoptera : Bombidae). J. Kansas entomol. Soc., 47 (3) : 396-404.

KAissling K. E., RenNer M., 1968. - Antennal receptors for queen substance and scent gland odour in Honey-bees. Z. vergl. Physiol., 59 : 357-361.

Kullenberg B., Bergstrom G., Stallberg-Stenhagen S., 1970. - Volatile components of the cephalic marking secretion of male Bumble bees. Acta Chem. Scand., 24 : 1481-1483.

Kullenberg B., Bergstrom G., Bringer B., Carlberg B., Cederberg B., 1973. - Observations on scent marking by Bombus Latr. and Psithyrus Lep. males (Hym. Apidae) and localization of site of production of the secretion. Zoon suppl., 1 : 23-29.

LACHER V., 1964. - Elektrophysiologische Untersuchungen an einzelnen Rezeptoren für Geruch, Kohlendioxyd, Luftfeuchtigkeit und Temperatur auf den Antennen der Arbeitsbiene und der Drohne (Apis mellifica L.) Z. vergl. Physiol., 48 : 587-623.

LaCher V., SChNeider D., 1963. - Elektrophysiologischer Nachweis der Riechfunktion von Porenplatten (sensilla placodea) auf den Antennen der Drohne und der Arbeitsbiene (Apis mellifica). Z. vergl. Physiol., 47 : 274-278.

Masson C., 1972. - Organisation sensorielle des principales articulations de l'antenne de la fourmi Camponotus vagus Scop. (Hymenoptera, Formicidae). Z. Morphol. Tiere, 73 : 343-359.

Masson C., 1973. - Contribution à l'étude du système antennaire chez les fourmis. Approche morphologique, ultrastructurale et électrophysiologique du système sensoriel. Thèse. Fac. Sci. Nat. Univ. Provence. Marseille. $332 \mathrm{p}$.

Masson C., 1974. - Quelques données sur l'ultrastructure de récepteurs gustatifs de l'antenne de la fourmi Camponotus vagus Scop. (Hymenoptera, Formicinae). Z. Morphol. Tiere, 77 : 235-243.

Masson C., Gabouriaut D., 1973. - Ultrastructure de l'organe de Johnston de la fourmi Camponotus vagus Scop. (Hymenoptera, Formicinae). Z. Zellforsch., 140 : 39-75.

Masson C., Brossut R., 1981. - La communication chimique chez les insectes. La Recherche, 121:406416.

Plowright R.C., Pendrel B. A., 1977. - Larval growth in bumble bees (Hymenoptera, Apidae). Can. Ent., 109 : 967-973.

Röseler P. F., 1975. - The role of juvenile hormone in regulating the fertility of the worker caste of the bumblebee, Bombus terrestris (L.). Verh. Dtsch. Zool. Ges. : 124.

RöSElER P.F., 1977. - Juvenile Hormone control of oögeneus in Bumble bee workers, Bombus terrestris. J. Insect Physiol., 23 : 985-992.

Röseler P. F., Röseler I., 1974. - Morphologische und physiologische Differenzierung der Kasten bei den Hummelarten Bombus hypnorum (L.) und Bombus terrestris (L.) Zool. Jb. Physiol., 78 : 175 198. 
SChNEIDER D., Steinbrecht R. A., 1968. - Checklist of insect olfactory sensilla. Symp. Zool. Soc. London, 23 : 279-297.

Sulfer E. H., 1961. - The fine structure of Insect sense organs. Intern. Rev. Cytol., 11 : 125-129.

SLIFER E. H., SEKhoN S. S., 1960. - The fine structure of the plate organs on the antenna of the Honeybee, Apis Mellifera Linnaeus. Exp. Cell. Res., 19 : 410-414.

Slifer E. H., SEKhon S. S., 1961. - Fine structure of the sense organs on the antennal flagellum of the Honeybee, Apis mellifera Linnaeus. J. Morphol., 109 (3) : 351-362 + pl.

Standifer L. N., Hull H. M., Bleckmann C. A., 1978. - Worker Honeybee antenna and mandible, seen through the scanning electron microscope. Bee World, 59 (3) : 101-104.

Svensson Bo. G., 1977. - Pheromones in male Bumblebees. A Taxonomical tool, exemplified by Pyrobombus lapponicus Fabr. (Hym. Apidae). Proc. 8th Int. Congr. I.U.S.S.I. 1977 : 317.

SVENSSON Bo. G., 1980. - Species isolating mechanisms in male bumble bees (Hymenoptera, Apidae). Acta Universitatis Upsaliensis : 549.

Van Honk C., Velthuis H., Roseler P. F., 1978. - A sex pheromone from the mandibular glands in Bumblebee queens. Experientia, $34: 838$.

Van Honk C., Velthuis H., Röseler P., Malotaux M., 1980. - The mandibular glands of Bombus terrestris queens as a source of queen pheromones. Entomol. exp. appl., 28 : 191-198.

VARESCHI E., 1971. - Duftunterscheidung bei der Honigbiene - Einzelzell-Ableitungen und Verhaltensreaktionen. Z. vergl. Physiol., $75: 143-173$.

WhiteHEAD A. T., LARSEN J. R., 1976. - Ultrastructure of the contact chemoreceptors of Apis mellifera L. (Hymenoptera : Apidae). Int. J. Insect Morphol. and Embryol., 5 (4/5) : 301-315. 
Annexe: Estimation de la surface antennaire étudiée.

a) Modèle géométrique pour les articles II à $I X$ du funicule (II à X pour le mâle).

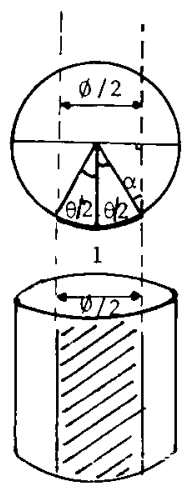

L'article antennaire étant assimilé à un cylindre de hauteur $\mathrm{h}$ et de diamètre $\varnothing$, sa surface est $\pi \cdot \varnothing \cdot \mathrm{h}$.

Soit 1 , la longueur d'un arc de cercle pour un angle $\theta$ (en degrés) :

$\sin \alpha=\varnothing / 4 / \varnothing / 2=\frac{1}{2} \Rightarrow \alpha=30^{\circ}$

$$
1=\frac{\pi \varnothing / 2 \theta}{180}
$$

$\sin \alpha=\sin \varnothing / 2 \quad \Rightarrow \theta=60^{\circ}$

$$
\Rightarrow 1=\frac{\pi \cdot \varnothing / 2 \cdot 60}{180}=\frac{\pi \cdot \varnothing / 2}{3}
$$

La surface hachurée (correspondant à un arc de cercle l) est de $\frac{\pi \cdot \varnothing / 2}{3} \cdot$ h et représente donc $1 / 6$ de la surface de l'article, $\pi \cdot \varnothing \cdot h$.

b) Modèle géométrique pour l'article distal.

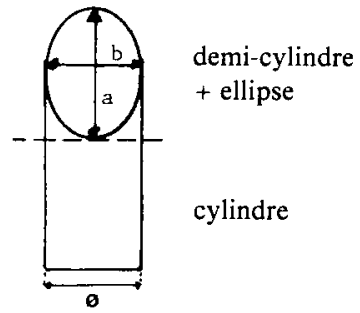

vue face inférieure

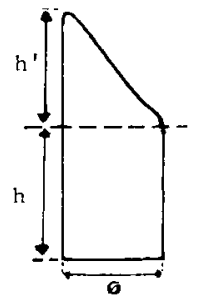

$\varnothing$ : diamètre

h : hauteur de la partie cylindrique

$h^{\prime}$ : hauteur de la partie apicale

a : petit axe de l'ellipse

b : grand axe de l'ellipse

vue latérale

Les mesures faites sur les photographies permettent de poser les hypothèses suivantes :

$$
\begin{aligned}
& \mathrm{a} \simeq \varnothing \\
& \mathrm{h}=\mathrm{h}^{\prime} \\
& \mathrm{b}^{2}=\mathrm{h}^{\prime 2}+\varnothing^{2}
\end{aligned}
$$

Surface de la partie cylindrique :

$$
\mathrm{s}_{1}=\pi \cdot \varnothing \cdot \mathrm{h}
$$

Surface de la partie apicale :

$$
s_{2}=\frac{\pi \cdot \varnothing}{2}\left(h^{\prime}+\frac{1}{2} \sqrt{h^{\prime 2}+\varnothing^{2}}\right) .
$$

Surface étudiée sur l'article distal :

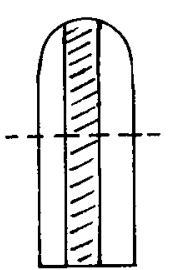

$$
\mathbf{S}=\mathbf{s}_{1}+\mathbf{s}_{2} \text {. }
$$

D'après les calculs effectués, la surface $S$ hachurée correspond à 16,7 à $18,2 \%$ de la surface totale de l'article. 